\title{
Analytical and quality control validation and assessment of field performance of a point-of-care chemistry analyzer for use in the white rhinoceros
}

\author{
Emma H. Hooijberg ${ }^{1, *}$ \\ Gerhard Steenkamp ${ }^{1}$ \\ Jacques P. du Preez ${ }^{1}$ \\ Amelia Goddard ${ }^{1}$
}

\footnotetext{
${ }^{1}$ Department of Companion Animal Clinical Studies, Faculty of Veterinary Science, University of Pretoria, Pretoria, South Africa
}

${ }^{*}$ Correspondence: Emma H. Hooijberg, Department of Companion Animal Clinical Studies, Faculty of Veterinary Science, Private Bag X04, Onderstepoort, Pretoria, 0110, South Africa Email: emma.hooijberg@up.ac.za

\begin{abstract}
Background: A chemistry point-of-care analyzer would be useful for evaluating injured wildlife, particularly white rhinoceros (Ceratotherium simum) that survive poaching attempts. The IDEXX VetTest ${ }^{\circledR}$ could be suitable, but species-specific validation, development of a statistical quality control (QC) strategy and evaluation under field conditions is necessary.

Objectives: The objectives were to 1) validate the VetTest for the white rhinoceros, 2) perform QC validation on the VetTest and generate a statistical QC strategy and 3) apply this QC strategy to monitor performance under typical field conditions.
\end{abstract}


Methods: Differences between white rhinoceros heparin plasma and serum, short-term imprecision and reportable range using rhinoceros plasma and long-term imprecision using commercial quality control material (QCM) was assessed against prescribed total allowable error $\left(\mathrm{TE}_{\mathrm{a}}\right)$ for up to 15 analytes. QC validation was performed using data from the long-term imprecision study and $\mathrm{TE}_{\mathrm{a}}$. A QC strategy using QCM was developed and used to monitor performance under field conditions.

Results: Imprecision was acceptable for all analytes except for ALP, ALT and AST at low activities. The reportable range for AST and LDH differed from the manufacturer's specifications. Eleven analytes were suitable for statistical QC using the $1_{3 \mathrm{~s}}$ rule, three using the $2_{s}$ rule; ALP was not suitable. In the field, observed error was less than $\mathrm{TE}_{\mathrm{a}}$ for all fifteen analytes and the sigma metric was $>3.0$ for twelve analytes.

Conclusions: The VetTest is suitable for use in the white rhinoceros. Statistical QC is possible for most analytes and useful for evaluation of field performance.

\section{Keywords}

Ceratotherium simum, dry chemistry, total allowable error, VetTest, wildlife

\section{Introduction}

From 2014-2015, 2390 rhinoceros (mainly white rhinoceros) were killed through poaching in South Africa. ${ }^{1}$ Some animals survive poaching attempts and require veterinary care for their injuries. ${ }^{2}$ Clinical pathology plays an important role in the initial evaluation and ongoing monitoring of these animals; however, the delay between blood sampling and subsequent analysis at a reference laboratory can be up to 24 hours. ${ }^{3}$ These compromised animals generally need to be chemically immobilized each time a veterinary procedure (including blood sampling) is performed, which is associated with the risk of respiratory depression, 
hypertension and renarcotization. ${ }^{4}$ A patient-side point of care analyzer (POCA) would provide immediate clinical information, limit immobilization events and decrease the risk of preanalytical errors associated with sample transport. Most POCAs used in veterinary practice are only validated for use in domesticated animals in a stable environment such as a veterinary practice laboratory. Before a POCA can be used for white rhinoceros, the analyzer must be validated for the species in question. ${ }^{5}$ Furthermore, recent guidelines published by the American Society for Veterinary Clinical Pathology (ASVCP) have emphasized the need for a quality control (QC) strategy for POCAs. ${ }^{6}$ Monitoring analyzer performance becomes particularly important when considering that a POCA used out in the field for wildlife is subject to a set of challenges not encountered in a stable practice or laboratory environment. These include factors like varying weather conditions, uneven roads, inconsistent power supply and dust. Although POCAs are commonly used for field work on non-domesticated animals, validation studies have not evaluated stability of performance in the field. ${ }^{7}$ The aims of this study were to (1) perform analytical validation of a POCA for use in the white rhinoceros (Ceratotherium simum), (2) to perform QC validation and formulate a statistical QC strategy for the POCA and (3) to assess the performance of the POCA under anticipated field conditions.

\section{Materials and methods}

\section{Analyzer}

The POCA evaluated in this study was the IDEXX VetTest ${ }^{\circledR}$ chemistry analyzer (IDEXX Laboratories, Inc., Westbrook, ME, USA). This analytical system uses reflectance photometry with dry-slide technology. ${ }^{8}$ Spectral analysis takes place using six lamps, each with a different wavelength and reactions take place at $37^{\circ} \mathrm{C}$. The ambient operating temperature range for the analyzer is $19-27^{\circ} \mathrm{C}$ and humidity range $30-50 \%$. Slides must be stored at $-18^{\circ} \mathrm{C}$ and can be used directly from the freezer. ${ }^{9} \mathrm{~A}$ maximum of 12 slides can be used per run. The VetTest used in this study was placed in the clinical pathology laboratory 
of the Onderstepoort Veterinary Academic Hospital under recommended operating conditions for the purpose of analyzer and QC validation. A full maintenance (including analysis of the manufacturer's quality control material) was performed on the analyzer before the study began. All analyses apart from those in the field study were carried out by one investigator $(\mathrm{EHH})$ after training by an IDEXX technician. A second investigator (JPP) assisted in the field study.

\section{Performance goals}

The ASVCP prescribed total allowable error $\left(\mathrm{TE}_{\mathrm{a}}\right)$ goals were used for both the method validation and the QC validation. ${ }^{10}$ For imprecision studies, ASVCP guidelines state that the imprecision, represented by the $C V$, should be less than the $\mathrm{TE}_{\mathrm{a} \cdot{ }^{11}}$ However, the total observed error $\left(\mathrm{TE}_{\mathrm{obs}}\right)$ should also be less than $\mathrm{TE}_{\mathrm{a}} \cdot{ }^{11} \mathrm{TE}_{\mathrm{obs}}$ is calculated by multiplying the CV by a factor of two, therefore the requirement for the imprecision studies here was that $\mathrm{CV}<0.5 \mathrm{TE}_{\mathrm{a}}$, in order to fulfill the criteria of $\mathrm{TE}_{\mathrm{obs}}<\mathrm{TE}_{\mathrm{a}} \cdot{ }^{10}$

\section{Method validation}

\section{Analytes and samples}

Fifteen analytes were evaluated: albumin (ALB), ALP, ALT, AST, total calcium (CA), CK, creatinine (CREA), GGT, glucose (GLU), lactate (LAC), LDH, magnesium (MG), inorganic phosphate (PHOS), total protein (TP) and urea. The analytical methods are shown in Table S1. ${ }^{8,9}$ Two types of sample material were used: an assayed human quality control material (QCM) (Bio-Rad Liquid Assayed Multiqual Level 1 and 2, Lot 45701/45702, Bio-Rad Laboratories Inc., Hercules, CA, USA) and samples from white rhinoceros. The rhinoceros samples had been collected previously for other studies and included blood from both healthy animals, immobilized for the purposes of translocation or preventative dehorning, and from clinically ill animals. Blood was collected from the auricular vein directly into serum and heparin tubes (BD Vacutainer, Becton and Dickinson, Plymouth, United Kingdom), 
stored in a cooler box with ice packs and centrifuged within 24 hours; serum and heparin plasma were aliquoted and stored at $-80^{\circ} \mathrm{C}$. Samples were up to three years old. Results from previous analyses of these samples on the laboratory's wet chemistry analyzer (Cobas Integra 400 Plus, Roche Diagnostics Ltd., Rotkreuz, Switzerland) were used to guide sample selection for the various experiments. Samples were excluded if gross hemolysis, lipemia or icterus was present. Approval to use the samples was granted by the University of Pretoria Animal Ethics Committee (V042-15).

\section{Comparison of serum versus heparin plasma}

Twenty paired serum and heparin plasma samples were used. Paired samples were thawed simultaneously; a panel of all analytes apart from LAC was measured on serum first followed by plasma for ten paired samples, and vice versa for the next ten samples.

\section{Short-term imprecision}

A high and a low pool was created for each analyte using white rhinoceros plasma. Pools were kept at room temperature after being made up and were used within 12 hours. Twenty measurements were performed for each analyte on each pool in two runs consisting of ten analyses each, with the second run immediately following on from the first.

\section{Long-term imprecision}

The long term imprecision study was carried out by running a panel containing all analytes on two levels of QCM once daily. Twenty such measurements were performed over a period of 31 days. Slides were not inserted in a particular order and a batch of 12 slides followed by a batch of three were used to complete the panel of 15 analytes. No results were obtained on two occasions for some analytes due to a slide spotting error and the missing data was obtained by running an extra panel for these analytes on day 31. The same QCM lot was used for all runs and the material was handled according to the manufacturer's recommendations. 
Reportable range

Evaluation of linearity and reportable range was carried out using rhinoceros plasma for AST, CK, LAC, LDH and TP. For each analyte, samples with a known high concentration were analyzed once in order to ensure that the analyte was within the reportable range. If this was successful, a further analysis in duplicate was immediately carried out to determine the mean analyte concentration; this sample was designated as level 5 . If the result was outside of the measurement range, distilled water was used to dilute the sample in a ratio of 1:2 and remeasured. Dilution and analysis were continued until a result was achieved, at which point a further duplicate analysis was carried out and the sample designated as level 5. A dilution series was prepared using distilled water (level 1 blank) and level 5 in ratios of 3:1 (level 2), 1:1 (level 3) and 1:3 (level 4). Levels 1 to 4 were then analyzed in triplicate. Statistical analysis and calculations

For the serum and plasma comparison, data were first tested for normality using the Kolmogorov-Smirnov test. For non-parametric data (ALP, ALT, CA, CK) the median and interquartile range were calculated and the difference between serum and plasma was assessed using the Wilcoxon matched-pair signed rank test. For data with a normal distribution, the mean and 95\% confidence intervals were calculated and the paired t-test was used to assess differences between serum and plasma. The difference between the mean or median was calculated as a percentage of the value for serum for each analyte and compared to $\mathrm{TE}_{\mathrm{a}}$. The Spearman's correlation coefficient rho $(r)$ was also calculated in order to assess the association between serum and plasma results. Level of significance was set at $P<.05$.

For both imprecision studies, the CV for each analyte, expressed in percentage, was calculated by dividing the SD by the mean multiplied by 100 for each pool or level. For the reportable range study, means were calculated from the triplicate measurements and plotted against target values of the dilution series. The resulting graph was inspected visually for linearity over the range of values and the slope and intercept calculated using ordinary least squares regression analysis. 
The programs and statistical tools used were Microsoft Excel spreadsheets (Microsoft Corp., Redmond, WA, USA) and SPSS version 22.0 (IBM Corp., Armonk, NY, USA).

\section{QC Validation}

QC validation was performed using the CVs obtained from the long-term imprecision study. As the supplier of the QCM did not supply target values for this analyzer, a useful estimate of bias for the purpose of QC validation could not be calculated and was set at zero for these calculations. $T E_{\mathrm{obs}}$ for each analyte for each level of QCM was calculated as ${ }^{10}$ $\mathrm{TE}_{\mathrm{obs}}(\%)=2 \mathrm{CV}$

The sigma metric $(\sigma)$ was calculated as ${ }^{12}$

$\sigma=\mathrm{TE}_{\mathrm{a}}(\%) / \mathrm{CV}(\%)$

The selection of appropriate control rules was performed in two steps. First, the $\mathrm{TE}_{\mathrm{a}}$ and $\mathrm{CV}$ for each analyte were matched to two simple control rules using a table from a recent publication which was formulated in order to assist in selection of appropriate control rules for in-clinic analyzers. ${ }^{13}$ This table provides for the use of either a $1_{3 \mathrm{~s}}$ rule with $n=1$, probability of error detection $\left(\mathrm{P}_{\mathrm{ed}}\right)$ of $\geq 85 \%$ and probability of false rejection $\left(\mathrm{P}_{\mathrm{fr}}\right)$ of $0 \%$, or a $1_{3 s}$ rule with $n=2, P_{\text {ed }}$ of $>90 \%$ and $P_{f r}=0 \%$. The $1_{3 s} n=1$ rule was used preferentially. When reviewing the suitability of the $1_{3 s} n=1$ rule, the CV corresponding with what was considered to be the more clinically relevant QCM level was used. Second, for analytes which could not be monitored by one of these rules, a sigma-metric QC design tool was used to identify candidate rules. ${ }^{12}$ Final rule selection was based on the criteria of $n \leq 2$, and that a simple rule was preferred over a multirule. 


\section{QC Strategy}

The $2_{s}$ or $3_{s}$ control rule limits were calculated from the SDs of the original set of QCM measurements. The mean values from the original 20 measurements served as the target values. A protocol for future statistical QC was developed based on the selected control rules and limits for the chosen levels. Levey-Jennings charts were created for each analyte.

\section{Field performance}

In order to simulate anticipated field conditions, the analyzer was placed in the closed back of a four-wheel drive vehicle which was driven around on dirt roads and uneven jeep tracks for four days in summer (November 2015). The vehicle was stationary during analyses, usually in the shade of a tree. Vehicle air-conditioning was left running at all times. Electricity was supplied via a 350W uninterruptible power supply unit (WAECO Sinepower MSI 412, Dometic WAECO International $\mathrm{GmbH}$, Emsdetten, Germany) from the vehicle's $12 \mathrm{~V}$ battery. The analyzer was placed inside a hard molded plastic airtight box with custom foam padding (Pelican Products Inc., Torrance, CA, USA). The analyzer was kept in this box during transport and taken out during measurement to facilitate operation of the ventilator fan. Slides were kept in a polystyrene cooler box with ice packs. Aliquots of QCM were placed frozen into this box at the start of the trip and thawed as needed. Ten sets of QCM measurements were carried out according to the QC strategy over four days and results of the QC analyses were recorded on the bespoke Levey-Jennings charts. The following were additionally recorded for each analysis: cooler box temperature, ambient temperature in the back of the vehicle, ambient outside temperature, any analyzer warnings. Temperatures were measured using digital thermometers with or without a probe. The analyzer was checked at the end of each day for dust inside the rotor cover and dusted if necessary. The mean and SD of the QC results from the field performance study $\left({ }_{\mathrm{fp}}\right)$ was used to calculate the $\mathrm{CV}_{\mathrm{fp}}$, bias $\mathrm{fp}, \mathrm{TE}_{\mathrm{fp}}$ and $\sigma_{\mathrm{fp}}$ as follows ${ }^{10}$

$\operatorname{Bias}_{\mathfrak{f p}}(\%)=\left(\operatorname{target}-\mathrm{mean}_{\mathrm{fp}}\right) / \operatorname{target} \times 100$ 
$\mathrm{TE}_{\mathrm{fp}}(\%)=2 \mathrm{CV}_{\mathrm{fp}}+$ bias $_{\mathrm{fp}}$

$\sigma_{\mathrm{fp}}=\left(\mathrm{TE}_{\mathrm{a}}-\mathrm{bias}_{\mathrm{fp}}\right) / C V_{\mathrm{fp}}$

\section{Results}

\section{Method validation}

Heparin plasma versus serum

Results are presented in Table 1. MG was significantly higher and PHOS lower in plasma compared to serum. The percentage difference between the medians or means obtained was within the total allowable error for all analytes. There was only moderate correlation for ALT, AST, LDH and TP. ${ }^{14}$

Short-term imprecision

The CVs varied between low and high species-specific pools, but were below $7 \%$ for all analytes except the low pools for ALT and AST (Table 2). The CV for the low pool of ALT and AST exceeded $0.5 \mathrm{TE}_{\mathrm{a}}$; all other CVS met the performance goals.

Long-term imprecision

Slide spotting failures occurred on two occasions, on day 3 with level 1 (ALB, AST, GLUC) and day 18 with level 2 (ALB, ALP, ALT). Each time these were the three slides in the second batch and the failure was due to inadequate sample material in the cup. Imprecision was $<10 \%$ for all analytes except for ALP and AST level 1, where imprecision was $>0.5 \mathrm{TE}_{\mathrm{a}}$ (12.5\%). Results are presented in Table 2. 
Table 1: Results of comparing white rhinoceros serum to heparin plasma

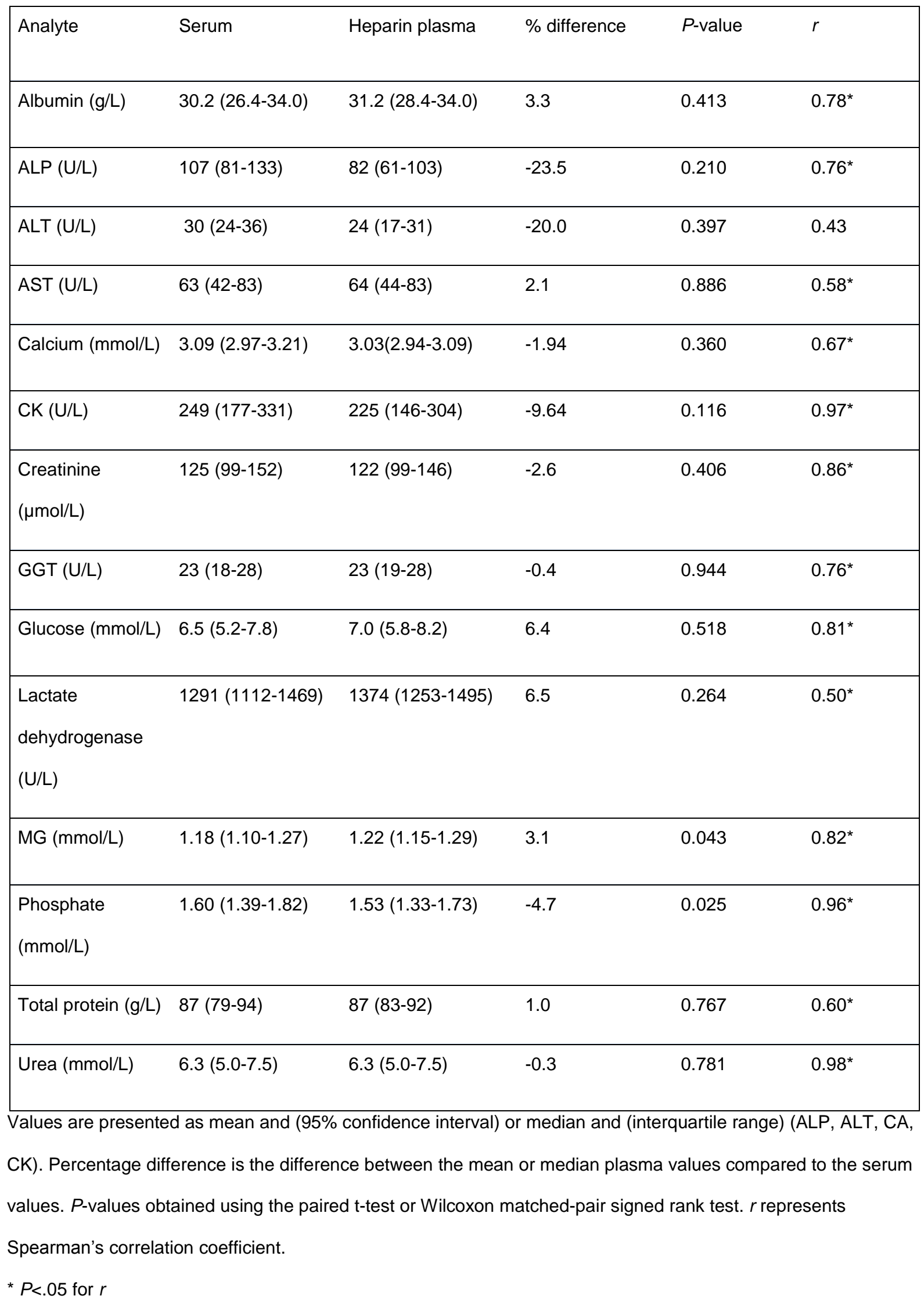


Table 2: Results of the short-term and long-term imprecision studies. Short-term imprecision was determined from white rhinoceros heparin plasma pools while long-term imprecision was determined from quality control material.

\begin{tabular}{|c|c|c|c|c|}
\hline \multirow[t]{2}{*}{ Analyte } & \multicolumn{2}{|c|}{ Short-term imprecision } & \multicolumn{2}{|c|}{ Long-term imprecision } \\
\hline & Mean & CV (\%) & Mean & CV (\%) \\
\hline \multirow[t]{2}{*}{ Albumin (g/L) } & 24 & 0.9 & 30 & 2.5 \\
\hline & 34 & 1.5 & 37 & 3.7 \\
\hline \multirow[t]{2}{*}{ ALP (U/L) } & 92 & 6.7 & 108 & 14.1 \\
\hline & 279 & 4.3 & 228 & 9.3 \\
\hline \multirow[t]{2}{*}{$\operatorname{ALT}(\mathrm{U} / \mathrm{L})$} & 22 & 34.7 & 94 & 8.6 \\
\hline & 236 & 2.2 & 179 & 3.9 \\
\hline \multirow[t]{2}{*}{ AST (U/L) } & 41 & 23.2 & 107 & 12.8 \\
\hline & 837 & 3.1 & 278 & 3.4 \\
\hline \multirow[t]{2}{*}{ Calcium (mmol/L) } & 3.01 & 1.8 & 2.77 & 1.3 \\
\hline & 3.38 & 1.0 & 3.35 & 1.1 \\
\hline \multirow[t]{2}{*}{ CK (U/L) } & 108 & 3.5 & 208 & 5.0 \\
\hline & 990 & 4.1 & 395 & 5.1 \\
\hline \multirow[t]{2}{*}{ Creatinine $(\mu \mathrm{mol} / \mathrm{L})$} & 92 & 2.9 & 198 & 3.8 \\
\hline & 319 & 1.0 & 709 & 1.7 \\
\hline \multirow[t]{2}{*}{ GGT (U/L) } & 24 & 4.4 & 112 & 1.2 \\
\hline & 65 & 1.4 & 138 & 1.2 \\
\hline \multirow[t]{2}{*}{ Glucose (mmol/L) } & 4.55 & 2.0 & 6.80 & 1.5 \\
\hline & 12.39 & 1.0 & 20.04 & 1.4 \\
\hline Lactate (mmol/L) & 6.03 & 1.8 & 3.19 & 2.7 \\
\hline
\end{tabular}




\begin{tabular}{|c|c|c|c|c|}
\hline & \\
\hline & 11.82 & 0.9 & 5.55 & 1.6 \\
\hline \multirow{2}{*}{$\begin{array}{l}\text { Lactate } \\
\text { dehydrogenase } \\
\text { (U/L) }\end{array}$} & 1269 & 3.7 & 425 & 7.2 \\
\hline & 1750 & 6.7 & 1094 & 4.2 \\
\hline \multirow{2}{*}{$\begin{array}{l}\text { Magnesium } \\
(\mathrm{mmol} / \mathrm{L})\end{array}$} & 1.14 & 1.6 & 1.10 & 1.7 \\
\hline & 1.32 & 2.2 & 1.55 & 2.0 \\
\hline \multirow[t]{2}{*}{ Phosphate (mmol/L) } & 0.92 & 1.8 & 1.56 & 5.0 \\
\hline & 2.22 & 2.2 & 2.52 & 1.3 \\
\hline \multirow[t]{2}{*}{ Total protein (g/L) } & 60 & 1.2 & 57 & 2.0 \\
\hline & 110 & 1.2 & 69 & 2.2 \\
\hline \multirow[t]{2}{*}{ Urea (mmol/L) } & 4.7 & 2.4 & 13.8 & 3.2 \\
\hline & 20.7 & 1.7 & 22.3 & 2.6 \\
\hline
\end{tabular}

$\mathrm{CV}=$ coefficient of variation

\section{Reportable range}

All five analytes showed a linear range under dilution, with linear correlation coefficients of 0.98 for AST and $\geq 0.99$ for CK, LAC, LDH and TP. The analytical range, slope and intercept of the regression lines are shown in Table 3. Level 1 and level 5 values were close to the manufacturer's reportable range for $\mathrm{CK}, \mathrm{LAC}$ and $\mathrm{TP} .{ }^{9}$ The highest measurable activity was $885 \mathrm{U} / \mathrm{L}$ for AST (reported range 0-1083 U/L). The measured analytical range for LDH was 117-1781 U/L, in contrast to the manufacturer's reported range of $50-2800 \mathrm{U} / \mathrm{L}^{9}$ 
Table 3: Results of the linearity study for five analytes in white rhinoceros plasma obtained by regression analysis

\begin{tabular}{|lllll|}
\hline Analyte & Analytical range & $r$ & Intercept & Slope \\
\hline AST $(\mathrm{U} / \mathrm{L})$ & $0-885$ & 0.98 & $-21(-178-136)$ & $0.96(0.67-1.25)$ \\
\hline CK $(\mathrm{U} / \mathrm{L})$ & $0-1522$ & 0.99 & $90(-153-334)$ & $1.00(0.74-1.24)$ \\
\hline Lactate (mmol/L) & $0-10.53$ & $>0.99$ & $0.45(-0.75-1.65)$ & $0.99(0.80-1.17)$ \\
\hline LDH (U/L) & & & $65(-78-207)$ & $0.99(0.86-1.11)$ \\
\hline Total protein (g/L) & $0-109$ & $>0.99$ & $4(-6-15)$ & $0.98(0.81-1.14)$ \\
\hline
\end{tabular}

$r$ represents the linear correlation coefficient. Results for the intercept and slope of the regression line are presented with $95 \%$ confidence intervals in parentheses.

\section{QC Validation}

Table 4 contains the $\mathrm{TE}_{\mathrm{obs}}$ and $\sigma$ values as well the selected $\mathrm{QC}$ rules with corresponding $P_{\text {ed }}$ and $P_{f r}$. TE $E_{o b s}$ was less than TE $E_{a}$ for all controls except ALP level 1 and AST level 1. A $\sigma$ value of $\geq 6.0$ was obtained for both $Q C$ levels for five analytes and for one $Q C$ level for six analytes. ALP had $\sigma<3.0$ for both QCM levels and ALT, AST and LDH had $\sigma<3.0$ for level 1. Six analytes were suitable for statistical QC using the $1_{3 \mathrm{~s}} \mathrm{n}=1$ rule at the clinically relevant QCM level. A further 5 analytes were suitable for statistical QC using the $1_{3 s} n=2$ rule. Statistical QC could be applied to LDH, TP and UREA using the $1_{2 s} n=2$ rule with a $P_{\text {ed }}$ of $>85 \%$; however, the $1_{2 s}$ rule is associated with a $P_{f d}$ of $9 \%$ for each measurement. ALP was not suitable for statistical QC using a $\mathrm{TE}_{\mathrm{a}}$ of $25 \%$; a $1_{2 \mathrm{~s}} \mathrm{n}=2$ rule gave a $\mathrm{P}_{\mathrm{ed}}$ of $30 \%$. 
Table 4: Total observed error, sigma metric and selected quality control rules for 15 analytes on the VetTest using two levels of quality control material (QCM). The more clinically relevant QCM level is bolded. The probability of error detection and false rejection for each rule are shown.

\begin{tabular}{|c|c|c|c|c|c|c|c|c|}
\hline Analyte & $\begin{array}{l}\text { QCM } \\
\text { Level }\end{array}$ & $\begin{array}{l}\mathrm{TE}_{\mathrm{obs}} \\
(\%)\end{array}$ & $\begin{array}{l}\text { Sigma } \\
\text { metric }\end{array}$ & $\begin{array}{l}\text { Suitable for } \\
1_{3 s} n=1 \\
\text { Ped }>85 \%\end{array}$ & $\begin{array}{l}\text { Suitable for } \\
1_{3 s} n=2 \\
\text { Ped }>90 \%\end{array}$ & $\begin{array}{l}\text { Rule } \\
\text { selected }\end{array}$ & $P_{e d}$ & $P_{f r}$ \\
\hline \multirow[t]{2}{*}{ Albumin } & 1 & 4.9 & 6.0 & No & Yes & $1-3 s n=2$ & $>90 \%$ & $0 \%$ \\
\hline & 2 & 7.4 & 4.1 & No & & & & \\
\hline \multirow[t]{2}{*}{ ALP } & 1 & 28.2 & 2.8 & No & & & & \\
\hline & 2 & 18.6 & 2.7 & No & No & $1-2 s \mathrm{n}=2$ & $30 \%$ & $9 \%$ \\
\hline \multirow[t]{2}{*}{ ALT } & 1 & 17.2 & 2.9 & No & & & & \\
\hline & 2 & 7.8 & 6.4 & No & Yes & $1-3 s \mathrm{n}=2$ & $>90 \%$ & $0 \%$ \\
\hline \multirow[t]{2}{*}{ AST } & 1 & 25.7 & 2.0 & No & & & & \\
\hline & 2 & 6.8 & 7.4 & No & Yes & $1-3 s n=2$ & $>90 \%$ & $0 \%$ \\
\hline \multirow[t]{2}{*}{ Calcium } & 1 & 2.6 & 7.7 & No & & & & \\
\hline & 2 & 2.2 & 9.7 & Yes & Yes & $1-3 s n=1$ & $>85 \%$ & $0 \%$ \\
\hline \multirow[t]{2}{*}{ CK } & 1 & 10.0 & 6.0 & No & & & & \\
\hline & 2 & 10.2 & 5.9 & No & Yes & $1-3 s \mathrm{n}=2$ & $>90 \%$ & $0 \%$ \\
\hline \multirow[t]{2}{*}{ Creatinine } & 1 & 7.7 & 5.3 & No & Yes & $1-3 s n=2$ & $>90 \%$ & $0 \%$ \\
\hline & 2 & 3.3 & 11.8 & Yes & & & & \\
\hline \multirow[t]{2}{*}{ GGT } & 1 & 2.5 & 16.7 & Yes & Yes & $1-3 s n=1$ & $>85 \%$ & $0 \%$ \\
\hline & 2 & 2.4 & 16.7 & Yes & & & & \\
\hline \multirow[t]{2}{*}{ Glucose } & 1 & 3.8 & 13.4 & Yes & Yes & $1-3 s n=1$ & $>85 \%$ & $0 \%$ \\
\hline & 2 & 2.8 & 14.3 & Yes & & & & \\
\hline
\end{tabular}




\begin{tabular}{|c|c|c|c|c|c|c|c|c|}
\hline \multirow[t]{2}{*}{ Lactate } & \multirow{2}{*}{$\begin{array}{l}1 \\
2\end{array}$} & \multirow{2}{*}{$\begin{array}{l}5.5 \\
3.1\end{array}$} & \multirow{2}{*}{$\begin{array}{l}14.8 \\
25.0\end{array}$} & \multicolumn{2}{|l|}{ Yes } & \multirow[b]{2}{*}{$1-3 s n=1$} & \multirow[b]{2}{*}{$>85 \%$} & \multirow[b]{2}{*}{$0 \%$} \\
\hline & & & & Yes & Yes & & & \\
\hline \multirow[t]{2}{*}{ LDH } & 1 & 14.3 & 2.7 & No & & & & \\
\hline & 2 & 8.5 & 4.8 & No & No & $1-2 s \mathrm{n}=2$ & $>90 \%$ & $9 \%$ \\
\hline \multirow[t]{2}{*}{ Magnesium } & 1 & 3.4 & 11.8 & Yes & Yes & $1-3 s n=1$ & $>85 \%$ & $0 \%$ \\
\hline & 2 & 3.9 & 10.0 & Yes & & & & \\
\hline \multirow[t]{2}{*}{ Phosphate } & 1 & 10.0 & 3.0 & No & & & & \\
\hline & 2 & 2.5 & 11.5 & Yes & Yes & $1-3 s n=1$ & $>85 \%$ & $0 \%$ \\
\hline \multirow[t]{2}{*}{ Total protein } & 1 & 4.1 & 5.0 & No & & & & \\
\hline & 2 & 4.5 & 4.5 & No & No & $1-2 s \mathrm{n}=2$ & $>90 \%$ & $9 \%$ \\
\hline \multirow[t]{2}{*}{ Urea } & 1 & 6.4 & 3.8 & No & & & & \\
\hline & 2 & 5.3 & 4.6 & No & No & $1-2 s n=2$ & $85 \%$ & $9 \%$ \\
\hline
\end{tabular}

$\sigma=$ sigma metric

$P_{\text {ed }}=$ probability of error detection

$\mathrm{P}_{\mathrm{fr}}=$ probability of false rejection

\section{QC Strategy}

The target values and rule limits for each analyte are shown in Table 5. Analytes monitored with QCM level 1 were ALB, ALP, ALT, AST, CK, CREA, GGT, GLU, LDH, MG, TP and UREA. Analytes monitored with QCM level 2 included ALB, ALP, ALT, AST, CA, CK, CREA, LAC, LDH, PHOS, TP and UREA 
Table 5: Quality control strategy for the VetTest using either one or two levels of quality control material, based on results of quality control validation.

\begin{tabular}{|c|c|c|c|}
\hline Analyte & QCM Level & Target & Limits \\
\hline \multirow[t]{2}{*}{ Albumin $(g / L)$} & 1 & 30 & $28-32$ \\
\hline & 2 & 37 & $33-42$ \\
\hline \multirow[t]{2}{*}{ ALP (U/L) } & 1 & 108 & $77-138$ \\
\hline & 2 & 228 & $186-271$ \\
\hline \multirow[t]{2}{*}{ ALT (U/L) } & 1 & 94 & $70-118$ \\
\hline & 2 & 179 & $158-200$ \\
\hline \multirow[t]{2}{*}{ AST (U/L) } & 1 & 107 & $66-148$ \\
\hline & 2 & 278 & $250-306$ \\
\hline Calcium (mmol/L) & 2 & 3.35 & $3.24-3.47$ \\
\hline \multirow[t]{2}{*}{ CK (U/L) } & 1 & 208 & $176-239$ \\
\hline & 2 & 395 & $335-456$ \\
\hline \multirow[t]{2}{*}{ Creatinine $(\mu \mathrm{mol} / \mathrm{L})$} & 1 & 198 & $175-221$ \\
\hline & 2 & 709 & $674-745$ \\
\hline GGT (U/L) & 1 & 112 & $108-116$ \\
\hline Glucose (mmol/L) & 1 & 6.80 & $6.49-7.10$ \\
\hline Lactate (mmol/L) & 2 & 5.55 & $5.3-5.8$ \\
\hline \multirow[t]{2}{*}{ LDH (U/L) } & 1 & 425 & $364-486$ \\
\hline & 2 & 1094 & $1001-1186$ \\
\hline Magnesium (mmol/L) & 1 & 1.10 & $1.04-1.15$ \\
\hline Phosphate (mmol/L) & 2 & 2.52 & $2.42-2.62$ \\
\hline Total protein (g/L) & 1 & 57 & $55-59$ \\
\hline
\end{tabular}




\begin{tabular}{|lccc|}
\cline { 2 - 4 } & 2 & 69 & $66-72$ \\
\hline Urea $(\mathrm{mmol} / \mathrm{L})$ & 1 & 13.8 & $12.9-14.7$ \\
\cline { 2 - 4 } & 2 & 22.3 & $21.1-23.5$ \\
\hline
\end{tabular}

$\mathrm{QCM}=$ quality control material

\section{Field performance study}

Outdoor temperatures ranged from $24.4-35.0^{\circ} \mathrm{C}$. Temperatures in the back of the vehicle ranged from $24.4-30.0^{\circ} \mathrm{C}$ and exceeded $27.0^{\circ} \mathrm{C}$ on three occasions. The temperature inside the cooler box ranged from $-4.7^{\circ}$ to $4.0^{\circ} \mathrm{C}$, with the temperatures increasing over the course of each day. The analyzer gave temperature warnings at the end of analysis when the ambient temperature exceeded $27^{\circ} \mathrm{C}$, but still delivered results. There was no visible dust seen inside the rotor cover. Results were outside of control limits for CREA and LDH once, and for AST, GGT and UREA twice. All these QC failures, except for one GGT measurement, were associated with a high temperature warning. There were multiple failures for TP (seven times for level 1, nine times for level 2) with results above the upper limit as shown in the Levey-Jennings charts in Figure 1. $T E_{f p}$ was less than $T E_{a}$ for all analytes. The sigma metric was $>3.0$ for all analytes except for ALP and UREA level 1 and TP both levels. These results are shown in Table S2. 


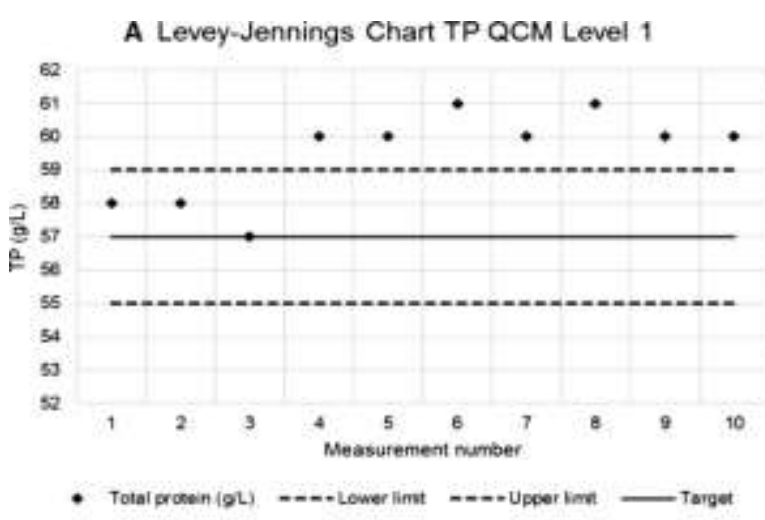

C Levey-Jennings Chart GLUC QCM Level 1

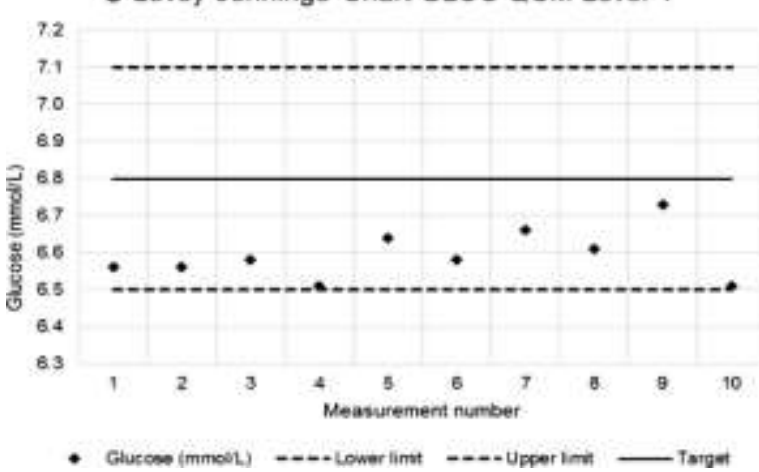

B Levey-Jennings Chart TP QCM Level 2

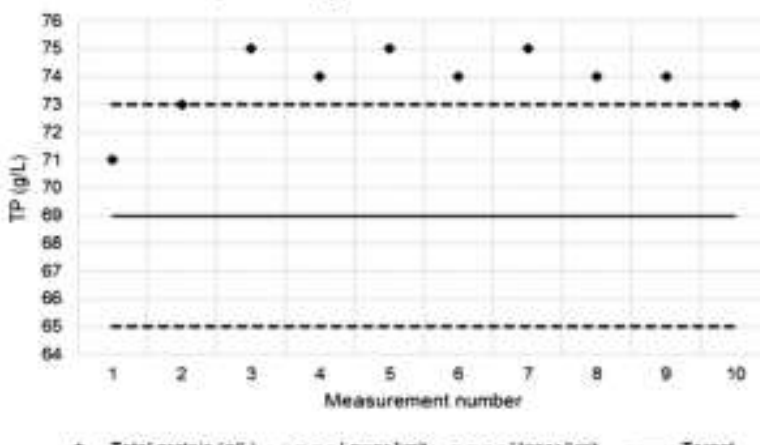

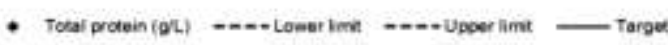

D Levey-Jennings Chart LAC QCM Level 2

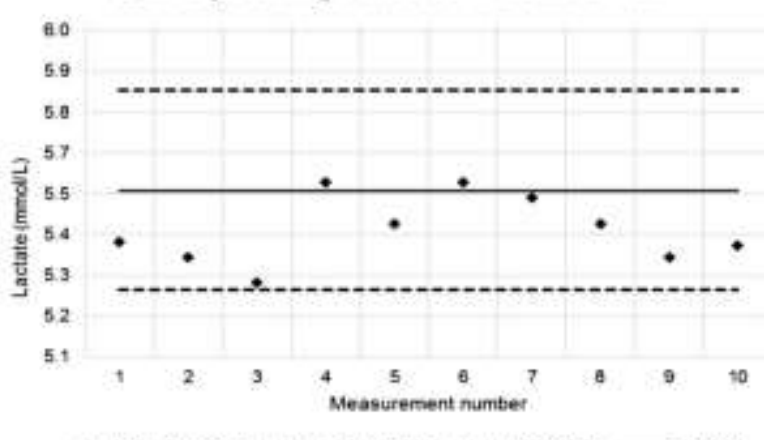

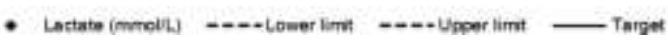

Figure 1. Levey-Jennings charts showing the results for total protein for two levels (A and B); and glucose (C) and lactate (D) for one level of quality control material measured ten times on the VetTest over four days under field conditions. The solid black line represents the target and the dotted lines represent the predetermined control limits. TP was out of control limits 7/10 times for level 1 and 9/10 times for level 2 . All results for glucose and lactate are within limits. All three analytes are measured by the $562 \mathrm{~nm}$ lamp.

\section{Discussion}

Overall, the POCA fulfilled most of the method validation requirements and can be used for the white rhinoceros. Application of ASVCP guidelines for quality control in POCAs based on QC validation was successfully applied. The resulting quality control strategy was used to assess performance of the analyzer in the field, with acceptable results.

The user manual for the VetTest states that serum and lithium heparin plasma may be used interchangeably for analytes examined in this study except lactate, but no further information is given. ${ }^{9}$ Lactate can be measured from heparin plasma if centrifuged and separated from red blood cells within five minutes of collection. ${ }^{9}$ This study revealed significant differences 
for MG and PHOS concentrations between heparin plasma and serum for the white rhinoceros as well as only moderate correlations for four other analytes (ALT, AST, LDH and TP). The differences found here could be method or species-related. A study comparing results for white rhinoceros heparin plasma and serum using another POCA found differences for ALP, AST, GGT, TP, BUN, CK and ALB, which differs from the findings here. ${ }^{15}$ Differences in MG and PHOS in heparin plasma versus serum have not been reported in dogs, cattle, horses or sheep. ${ }^{16-19}$ The reason for these findings here are unclear, however care should be taken in using plasma and serum interchangeably in this and other species. Using plasma rather than serum delivers faster results as the sample does not need to be left to clot before centrifugation. This is potentially critical when attending to an injured or immobilized white rhinoceros. Based on the findings presented here, all further experiments were conducted on heparin plasma and not serum.

White rhinoceros plasma was used for the short-term imprecision study in order to assess species-specific imprecision. A commercial liquid QCM was used in the long-term imprecision study and for QC validation, as in other studies, as this type of material is practical and commonly used for internal QC. ${ }^{13,20-22}$ Short-term imprecision was acceptable for most analytes, similar to results from other studies using equine serum and canine and pigeon plasma. . 23,24 The imprecision for AST of $23 \%$ and ALT of $35 \%$ at low activity levels was higher than half the $\mathrm{TE}_{\mathrm{a}}(12.5 \%)$, however this may be of little or no clinical significance at low levels of results. Previous studies reported an imprecision of $2-8 \%$ for AST at similar or higher activity levels but were performed on material from other species. ${ }^{8,23,24}$ None of these studies calculated the short-term imprecision of ALT on patient samples; however, a recent study found a CV of $7 \%$ for $\mathrm{ALT}$, using a commercial $\mathrm{QCM}$, at an activity of $37 \mathrm{IU} / \mathrm{L} .^{20}$ These rhinoceros-specific measures of imprecision reported in our study can be used to aid in interpretation of patient data in the future.

Long-term imprecision fell within performance goals for all analytes except for ALP and AST at the lower level. A long-term imprecision of $2-4 \%$ for ALP and $2-7 \%$ for AST has been 
reported for similar or slightly higher enzyme activities in some studies examining imprecision in this analyzer. ${ }^{8,20,24}$ The results for long-term imprecision in this and the aforementioned studies are in contrast to data obtained from this analyzer in use in veterinary practices, where imprecision was often much higher than obtained here. ${ }^{13}$ It is likely that the high imprecision observed in-practice is due to the low enzyme activities in these samples and is usually not clinically relevant. ${ }^{10}$

AST, CK, LAC, LDH and TP were chosen for the reportable range study as high levels had been noted while making up the pools for the imprecision study, and linearity up to the upper reported analytical limit is potentially of clinical importance. It was however not possible to obtain results for AST and LDH near the upper reported limits. No published study on reportable range for this analyzer on any material was found, and it is not possible to conclude whether the reported ranges for these analytes are inaccurate or whether there are interfering substances present in white rhinoceros plasma leading to these findings. As many injured white rhinoceros suffer from a myopathy with very high reported activities of muscle enzymes, it may be prudent for clinicians to perform a 1:4 or 1:9 dilution before running AST, $\mathrm{CK}$ and $\mathrm{LDH}^{3}$

Bias estimates for a POCA can firstly provide information for the assessment and monitoring of analytical performance and secondly be used to determine whether reference intervals derived for another method are valid. ${ }^{5}$ Initial species-specific analyzer performance was assessed here based on linearity and precision studies using white rhinoceros plasma. Bias is ideally calculated during instrument performance studies from a method comparison experiment, where the field method is compared to a gold standard method. ${ }^{5,25}$ This was not possible here due to the lack of a gold standard. A wet chemistry analyzer (Cobas Integra 400 Plus) is used in the authors' laboratory for routine rhinoceros samples, but this analyzer has not been validated for this species and is another field method. Results of a method comparison between the VetTest and Cobas Integra for ten of the analytes investigated here are detailed in a separate publication. ${ }^{26}$ Using bias obtained from comparison with a field 
method for quantification of $\mathrm{TE}_{\mathrm{a}}$ and $\mathrm{QC}$ validation can overestimate the error assigned to the comparative method and was not used in the calculations here. ${ }^{21}$ Bias can also be quantified using mean values provided for an assayed QCM as "true" values, however these were not available for this method and using the targets supplied for other methods is of questionable value. ${ }^{5}$ Method-specific target values were calculated from 20 measurements of the QCM and bias was subsequently measured for the purposes of analytical performance monitoring during the field performance study and incorporated into $T E_{o b s}$ calculations for that part of the study.

Designing a QC plan based on the use of validated control rules is regarded as the gold standard for interpreting QC data, even for veterinary POCAs. ${ }^{6,27}$ Furthermore, daily monitoring of POCA instrument performance is recommended by the ASVCP. ${ }^{6}$ The routine QC procedure prescribed for the VetTest by the manufacturer is an analysis once a month using QC material supplied by the manufacturer. ${ }^{9}$ A set of slides for six analytes, each testing one of the six lamps is also supplied. Results of the QC analysis are presented against a "reference range" which appears on the analyzer printout. Information concerning the derivation of this range, including the number of standard deviations it represents (two or three) is not available and no target mean values are provided. This strategy is not in line with current best practice guidelines and an alternative QC plan, following these guidelines, was therefore designed. ${ }^{6}$ Where possible, the $1_{3 \mathrm{~s}}$ rule was selected as this rule is considered to be most suitable for POCAs. ${ }^{6,13}$ The $1_{3 s}$ rule was suitable for use in $73 \%(11 / 15)$ of analytes. It has been suggested that POCAs should have $>75 \%$ of analytes controllable by the $1_{3 \mathrm{~s}}$ rule, with $\mathrm{P}_{\mathrm{fr}} \leq 5 \%$ and $\mathrm{P}_{\mathrm{ed}} \geq 85 \%$, in order to be fit for statistical quality control in a clinic environment. ${ }^{6}$ The use of other control rules requires the application of QC validation procedures, and was performed here. It was not possible to use statistical QC for ALP based on the data in this study, as a $\mathrm{P}_{\mathrm{ed}}$ of $30 \%$ is unacceptably low. The utility of running ALP on this analyzer is questionable if using a $\mathrm{TE}_{\mathrm{a}}$ of $25 \%$. Increasing the $\mathrm{TE}_{\mathrm{a}}$ is a possibility, and a 
new $\mathrm{TE}_{\mathrm{a}}$ could be calculated based on reference intervals and clinical decision limits. ${ }^{28}$ This data is however not available yet for white rhinoceros.

The $1_{2 s}$ rule provided an adequate $P_{\text {ed }}$ of $>85 \%$ for three analytes for which the $1_{3 s}$ rule was not suitable. Although the $1_{2 s}$ rule is associated with a high $P_{\mathrm{fr}}$, it is simpler for clinicians to apply than a multirule. Another option is to use a less stringent rule with more levels of QCM, but the cost of these additional QCM levels needs to be weighed up against the cost of repeating the analysis using the QCM levels already in use. QCM lot changes and recalibration through software updates could affect the control limits derived for this study and new data may need to be calculated in the event of a new lot or software update. ${ }^{6}$

Published information regarding evaluation of POCA performance in the field is available but scarce and focuses on method comparison between the POCA and a reference laboratory analyzer. ${ }^{7,29-31}$ The evaluation of bias however does not assess stability of the system over time. A human study evaluating a clinical chemistry analyzer in a military field laboratory followed a protocol advocated by the U.S National Committee for Laboratory Standards, in which precision, linearity and accuracy were monitored. ${ }^{29}$ This protocol was carried out in a pre-mobilization, mobilization and post-mobilization phase in that study. ${ }^{29}$ Monitoring of both accuracy and precision over time is more likely to reflect performance. The use of statistical QC facilitates measurement of both bias and imprecision against preset goals and was thus the objective evaluation tool used in this study. The analyzer generally performed well under field conditions, except when vehicle ambient temperature exceeded $27^{\circ} \mathrm{C}$. This is in line with the manufacturer's operating specifications and indicates the importance of measuring ambient temperature in the field, and keeping the operating environment as cool as possible. The cause for the TP QC failures was not clear and the positive bias present in the TP results represents a systematic error. TP is measured with the green $562 \mathrm{~nm}$ lamp, along with LAC and GLUC, which had good QC results with $\sigma>6.0$ for both (Fig 1). The TP slides were kept under the same conditions as the other slides. The manufacturer states that all slides can be recycled from cold storage to room temperature up to five times - some but 
not all of these slides would have undergone a temperature increase to a maximum of $4^{\circ} \mathrm{C}$ only once during the course of the experiment, therefore inaccuracies due to temperature changes seem unlikely. ${ }^{9}$ The same lot of TP slides was used for the long term imprecision and field performance and lot to lot variation can be ruled out. In a clinical scenario, the next step in troubleshooting would be to contact the manufacturer for further technical assistance, before running further patient samples. The formulation of bespoke Levey-Jennings charts and concurrent recording of environmental conditions assisted with trouble-shooting of QC failures. The Levey-Jennings charts in particular provide a user-friendly method of recording and assessment for operators not familiar with the concepts of QC.

The VetTest proved suitable for use in the white rhinoceros with heparin plasma samples, although the upper reportable limits for AST and LDH were much lower than those provided by the manufacturer for other species. Method comparison data and reference intervals for this POCA are presented in a separate study. ${ }^{26}$

This study provides an example of how QC validation and statistical QC can be applied to a POCA in line with ASVCP guidelines. ${ }^{6}$ Other aspects of quality assurance should however not be ignored. Operator training, formulation of standard operating procedures and comparability testing, for example, are all important elements of a total quality management strategy, and should be considered for this analyzer.

Providing clinical pathology data for wildlife means that patient-side analyzers may have to function in varying and challenging environmental conditions. The evaluation of performance using statistical QC shown here provides an example of how the stability of an analytical system can be evaluated under field conditions. Performing and evaluating QC each time the analyzer is used in the field will be vital to ensure the quality of patient results. 


\section{Acknowledgements}

The authors would like to recognize and thank Dr. Kathleen Freeman for her advice and guidance. This project was funded by the South African Veterinary Foundation, the Department of Companion Animal Clinical Studies of the University of Pretoria and Saving the Survivors. The quality control material used in the study was kindly donated by Bio-Rad Laboratories., Inc.

\section{References}

1. Department of Environmental Affairs RoSA. Minister Edna Molewa highlights progress in the fight against rhino poaching. 2016;

https://www.environment.gov.za/mediarelease/molewa_highlightsprogress_againstrhi nopoaching. Accessed March 22, 2016.

2. Hooijberg EH, Cray C, Du Preez JP, Steenkamp G, Goddard A. Routine inflammatory markers in an injured white rhinoceros (case report) [abstract]. Vet Clin Pathol. 2016;45:E12.

3. Du Preez JP, Tordiffe A, Meyer L, Steenkamp G. Comparing haematological and biochemical parameters of healthy and critically injured rhinoceroses. Poster presented at: Faculty Day; 20 August 2015, 2015; Faculty of Veterinary Science, University of Pretoria, South Africa.

4. Portas TJ. A review of drugs and techniques used for sedation and anaesthesia in captive rhinoceros species. Aust Vet J. 2004;82:542-549.

5. Flatland B, Friedrichs KR, Klenner S. Differentiating between analytical and diagnostic performance evaluation with a focus on the method comparison study and identification of bias. Vet Clin Pathol. 2014;43:475-486. 
6. Flatland B, Freeman KP, Vap LM, Harr KE. ASVCP guidelines: quality assurance for point-of-care testing in veterinary medicine. Vet Clin Pathol. 2013;42:405-423.

7. Stoot LJ, Cairns NA, Cull F, et al. Use of portable blood physiology point-of-care devices for basic and applied research on vertebrates: a review. Conserv Physiol. $2014 ; 2$.

8. Tschudi PR. Evaluation of the dry chemical analysis system VetTest 8008. Schweiz Arch Tierh. 1995;137:381-385.

9. IDEXX Laboratories. IDEXX VetTest@ Chemistry Analyzer Operator's Guide. 2010.

10. Harr KE, Flatland B, Nabity M, Freeman KP. ASVCP guidelines: allowable total error guidelines for biochemistry. Vet Clin Pathol. 2013;42:424-436.

11. Flatland B, Freeman KP, Friedrichs KR, et al. ASVCP quality assurance guidelines: control of general analytical factors in veterinary laboratories. Vet Clin Pathol. 2010;39:264-277.

12. Westgard JO. The sigma-metric QC-selection tool. Basic QC Practices Training in Statistical Quality Control for Medical Laboratories. Madison WI: Westgard QC; 2010:231-247.

13. Rishniw M, Pion PD, Maher T. The quality of veterinary in-clinic and reference laboratory biochemical testing. Vet Clin Pathol. 2012;41:92-109.

14. Zady MF. Z-stats 12 - Correlation and simple least squares regression (lesson). 2009; http://www.westgard.com/lession42.htm. Accessed December 2, 2015.

15. Mathebula N, Miller M, Buss $\mathrm{P}$, et al. Biochemical values in free-ranging white rhinoceros (Ceratotherium simum) in Kruger National Park, South Africa. J Zoo Wildlife Med. 2012;43:530-538.

16. Cerón JJ, Martínez-Subiela S, Hennemann C, Tecles F. The effects of different anticoagulants on routine canine plasma biochemistry. Vet J. 2004;167:294-301.

17. Mohri M, Allahyari L, Sardari K. Effects of Common Anticoagulants on Routine Plasma Biochemistry of Horse and Comparison with Serum. J Equine Vet Sci. 2007;27:313-316. 
18. Mohri M, Rezapoor H. Effects of heparin, citrate, and EDTA on plasma biochemistry of sheep: Comparison with serum. Res Vet Sci. 2009;86:111-114.

19. Mohri M, Shakeri H, Lotfollah Zadeh S. Effects of common anticoagulants (heparin, citrate and EDTA) on routine plasma biochemistry of cattle. Comp Clin Path. 2007;16:207-209.

20. Baral R, Morton J, Dhand N, Krockenberger M, Govendir M. Repeatability of results from three in-house biochemistry analyzers and a commercial laboratory analyzer used in small animal practice. Comp Clin Path. 2015;24:755-765.

21. Flatland B, Breickner LC, Fry MM. Analytical performance of a dry chemistry analyzer designed for in-clinic use. Vet Clin Pathol. 2014;43:206-217.

22. Farr AJ, Freeman KP. Quality Control Validation, Application of sigma metrics, and performance comparison between two biochemistry analyzers in a commercial veterinary laboratory. J Vet Diagn Invest. 2008;20:536-544.

23. Vereecken $M$, Vanrobaeys $M$, De Herdt $P$. Usefulness of two commercial analysers for plasma chemistry in pigeons. Vlaams Diergen Tijds. 2001;70:44-49.

24. Little CJ, Gettinby G, Downs J, Marshall CB. Consistency of results from the Vettest 8008 clinical biochemistry analyser. Vet Rec. 1992;130:419-424.

25. Jensen AL, Kjelgaard-Hansen M. Method comparison in the clinical laboratory. Vet Clin Pathol. 2006;35:276-286.

26. Hooijberg EH, Steenkamp G, Freeman KP, Buss P, Goddard A. Method comparison and generation of plasma biochemistry reference intervals for the white rhinoceros (Ceratotherium simum) on a point-of-care and wet chemistry analyzer. Submitted to Vet Clin Pathol 2016.

27. Westgard JO. Laboratory Analytical QC Plans and Quality Systems. Basic QC Practices Training in Statistical Quality Control for Medical Laboratories. Madison WI: Westgard QC; 2010:269-286. 
28. Lester S, Harr KE, Rishniw M, Pion P. Current quality assurance concepts and considerations for quality control of in-clinic biochemistry testing. J Am Vet Med Assoc. 2013;242:182-192.

29. Sullinger J, Garrett PE. A clinical chemistry analyzer evaluated by NCCLS guidelines for use in a military field laboratory unit. Clin Chem. 1989;35:2223-2226.

30. Lopes-Pereira Carlos M, Harun M, Schmidtova D, et al. Use of the dry chemistry "Reflotron" blood analyzer under outdoor-field conditions in veterinary medicine. cclm. 1996;34:231.

31. Stoskopf MK, Mulcahy DM, Esler D. Evaluation of a portable automated serum chemistry analyzer for field assessment of harlequin ducks, Histrionicus histrionicus. Vet Med Int. 2010;2010:418596. 


\section{Supplementary Tables}

Table S1: Analytes and analytical methods chosen for investigation in white rhinoceros on the IDEXX VetTest dry chemistry analyzer.

\begin{tabular}{|c|c|c|}
\hline Analyte & Method & Wavelength \\
\hline Albumin & $\begin{array}{l}\text { Bromocresol green dye-binding } \\
\text { method }\end{array}$ & $640 \mathrm{~nm}$ \\
\hline ALP & Kinetic, p-nitrophenol & $400 \mathrm{~nm}$ \\
\hline ALT & Kinetic, alanine and $\alpha$-ketoglutarate & $350 \mathrm{~nm}$ \\
\hline AST & $\begin{array}{l}\text { Kinetic, aspartate and } \alpha \text { - } \\
\text { ketoglutarate }\end{array}$ & $350 \mathrm{~nm}$ \\
\hline Bilirubin & Diazo method & $562 \mathrm{~nm}$ \\
\hline Calcium & Arsenazo III method & $680 \mathrm{~nm}$ \\
\hline Cholesterol & Enzymatic with cholesterol esterase & $562 \mathrm{~nm}$ \\
\hline Creatinine & $\begin{array}{l}\text { Enzymatic with creatine } \\
\text { amidinohydrolase }\end{array}$ & $640 \mathrm{~nm}$ \\
\hline CK & $\begin{array}{l}\text { Kinetic, creatine phosphate and } \\
\text { ADP }\end{array}$ & $680 \mathrm{~nm}$ \\
\hline GGT & $\begin{array}{l}\text { Kinetic with gamma-glutamyl-p- } \\
\text { nitroanilide cleavage }\end{array}$ & $400 \mathrm{~nm}$ \\
\hline Glucose & Glucose oxidase & $562 \mathrm{~nm}$ \\
\hline Magnesium & Formazan method & $640 \mathrm{~nm}$ \\
\hline
\end{tabular}




\begin{tabular}{|lll|}
\hline Phosphate & Phosphomolybdate method & $640 \mathrm{~nm}$ \\
\hline Lactate & Lactate dehydrogenase method & $562 \mathrm{~nm}$ \\
\hline LDH & Kinetic, pyruvate & $350 \mathrm{~nm}$ \\
\hline Total protein & Biuret method & $562 \mathrm{~nm}$ \\
\hline Triglycerides & Enzymatic with lipase & $562 \mathrm{~nm}$ \\
\hline Urea & Urease method & $640 \mathrm{~nm}$ \\
\hline
\end{tabular}


Table S2: Results of a field performance study on the VetTest where ten measurements of quality control material were made over four days and evaluated against control rules formulated during a quality validation process.

\begin{tabular}{|c|c|c|c|c|c|}
\hline Analyte & Lamp & QCM Level & $\begin{array}{l}\text { Number of } \\
\text { times QC } \\
\text { failed }\end{array}$ & $\mathrm{TE}_{\mathrm{fp}}(\%)$ & $\sigma_{\mathrm{fp}}$ \\
\hline \multirow[t]{2}{*}{ Albumin } & Red $640 \mathrm{~nm}$ & 1 & 0 & 7.2 & 5.3 \\
\hline & & 2 & 0 & 5.7 & 5.2 \\
\hline \multirow[t]{2}{*}{ ALP } & UV $400 \mathrm{~nm}$ & 1 & 0 & 21.1 & 2.5 \\
\hline & & 2 & 0 & 16.2 & 3.5 \\
\hline \multirow[t]{2}{*}{ ALT } & UV $350 \mathrm{~nm}$ & 1 & 0 & 17.9 & 3.1 \\
\hline & & 2 & 0 & 6.3 & 11.4 \\
\hline \multirow[t]{2}{*}{ AST } & UV $350 \mathrm{~nm}$ & 1 & 0 & 16.8 & 3.6 \\
\hline & & 2 & 2 & 15.3 & 4.0 \\
\hline Calcium & $\begin{array}{l}\text { Deep red } \\
680 \mathrm{~nm}\end{array}$ & 2 & 0 & 2.5 & 9.4 \\
\hline \multirow[t]{2}{*}{ CK } & Deep red & 1 & 0 & 5.8 & 11.0 \\
\hline & $680 \mathrm{~nm}$ & 2 & 0 & 10.1 & 6.4 \\
\hline \multirow[t]{2}{*}{ Creatinine } & Red $640 \mathrm{~nm}$ & 1 & 0 & 7.2 & 7.0 \\
\hline & & 2 & 1 & 5.2 & 11.9 \\
\hline GGT & UV $400 \mathrm{~nm}$ & 1 & 2 & 7.2 & 6.0 \\
\hline Glucose & $\begin{array}{l}\text { Green } 562 \\
\mathrm{~nm}\end{array}$ & 1 & 0 & 5.2 & 16.3 \\
\hline Lactate & $\begin{array}{l}\text { Green } 562 \\
\mathrm{~nm}\end{array}$ & 2 & 0 & 5.2 & 22.8 \\
\hline $\mathrm{LDH}$ & UV $350 \mathrm{~nm}$ & 1 & 0 & 10.4 & 4.5 \\
\hline
\end{tabular}




\begin{tabular}{|lllllc|}
\hline & & 2 & 1 & 8.8 & 4.6 \\
\hline Magnesium & Red 640 nm & 1 & 0 & 4.6 & 14.5 \\
\hline Phosphate & Red 640 nm & 2 & 0 & 2.4 & 12.4 \\
& & & & & \\
\hline Total protein & Green 562 & 1 & 7 & 8.9 & 2.5 \\
\cline { 2 - 6 } & $\mathrm{nm}$ & 2 & 9 & 9.5 & 2.3 \\
\hline Urea & Red 640 nm & 1 & 2 & 8.8 & 2.8 \\
\cline { 2 - 6 } & & 2 & 0 & 5.7 & 4.2 \\
\hline
\end{tabular}

QCM=quality control material

$\mathrm{QC}=$ quality control

$\mathrm{TE}_{\mathrm{fp}}=$ total observed error during field performance evaluation. $T E_{\mathrm{fp}}$ was less than allowable total error for all analytes

$\sigma_{\mathrm{fp}}=$ sigma metric derived from the field performance evaluation 